EPiC Series in Engineering
Volume 2, 2018, Pages 14-24
SUMO 2018- Simulating Autonomous
and Intermodal Transport Systems

\title{
Simulating a multi-airport region on different abstraction levels by coupling several simulations
}

\author{
Ulf Noyer ${ }^{1}$, Florian Rudolph ${ }^{1}$ and Martin Jung ${ }^{1}$ \\ ${ }^{1}$ Deutsches Zentrum für Luft- und Raumfahrt e.V. \\ Ulf.Noyer@dlr.de, Florian.Rudolph@dlr.de, M.Jung@dlr.de
}

\begin{abstract}
Airports build the pillar for international mobility and are core elements of intermodal traffic. They are interface between ground level transportation and air transport and commonly act as central points for logistics.

The DLR project "Optimode.net" investigates the interaction between relevant traffic modes and develops innovative approaches for integrated traffic management of ground and air level to extend the management of an airport not only to airport landside and to terminal processes but to go even further and incorporate feeder traffic in the management of airport processes. The realization of an integrated traffic planning is the foundation for several research questions, how the interaction between planes and ground transport can be improved. While the overall airport management benefits from more efficiency, travelers should be supported with a door-to-door management service.

The simulated scenario consists of four cities in a multi airport region. The simulation of the region includes three airports in different sizes with the simulation of the feeder traffic to the airports. All cities are connected by road and railroad and public ground level transport is available.

The procedure switching between a ground level vehicle and a plane needs several time consuming steps at the airport for individual passengers, including security checks, boarding and so on. Therefore, these steps are also simulated at the airport on a passenger level. Furthermore, the dispositioning of flights must be considered. On the ground level individual traffic, public road transport and railways are included in the simulation environment. In total, the complete simulation environment is built of nine simulation models with various abstraction levels. For the simulation of ground level vehicles in this environment, SUMO is used. This paper discusses the coupling and the data exchange between the different simulators and further focuses on the integration of SUMO in this setup.
\end{abstract}




\section{Introduction}

Airports are not just places where airplanes land and take-off but they are also natural interfaces between ground transport modes and air transport. In the door-to-door travel chain, it is not sufficient to just focus on a small part of the journey. Attention must be paid to the interlinking of transport modes in different phases of the journey. In recent years, substantial improvement could be generated in the field of airport management especially for landside and terminal processes (Milbredt, Rudolph, Grunewald, \& Christ, 2017). In a recent project called "Optimode.net", an innovative approach is being developed at the German Aerospace Center (DLR) to extend the concept of A-CDM (Airport Collaborative Decision Making) and TAM (Total Airport Management) not only to airport landside and terminal processes but to go even further and incorporate feeder traffic in the management of airport processes (Eurocontrol, 2012).

The integrated considerations of these airport processes need different approaches and are also examined by different working groups. Because of these reasons, specialized simulation environments must be used and coupled, what is discussed in the following sections. (Jung, Rudolph, Claßen, Pick, \& Noyer, 2017)

\section{Simulation environment}

This section discusses the simulation environment, which is modelled for the individual simulators. As it gives insight to the covered simulation setting and some details of the simulated aspects, it gives a high-level overview of the challenges.

\subsection{Scenario}

The simulation covers a full day from 00:00 to 23:59 in order to simulate typical traffic scenarios upcoming during a day. As traffic becomes typically quite low during the late night, more complex traffic situations arise during the day, it can be manipulated in all relevant states by the airport traffic management.

The simulation of the region includes three airports in different sizes with the simulation of the feeder traffic to the airport. Figure 1 shows the virtual multi airport region serving as the base structure for the simulation. There are four cities "A-Castle", "B-Field", "C-City" and "D-Town" being interconnected via road and railroad based on a real world scenario. A-Castle represents the largest city with over 1 million citizens and an international hub airport. B-Field has about 250,000 citizens and a small regional airport. C-City has about 500,000 citizens and a medium-sized international airport. D-Town lies between A-Caste and B-Field, has about 50,000 citizens and no airport. 


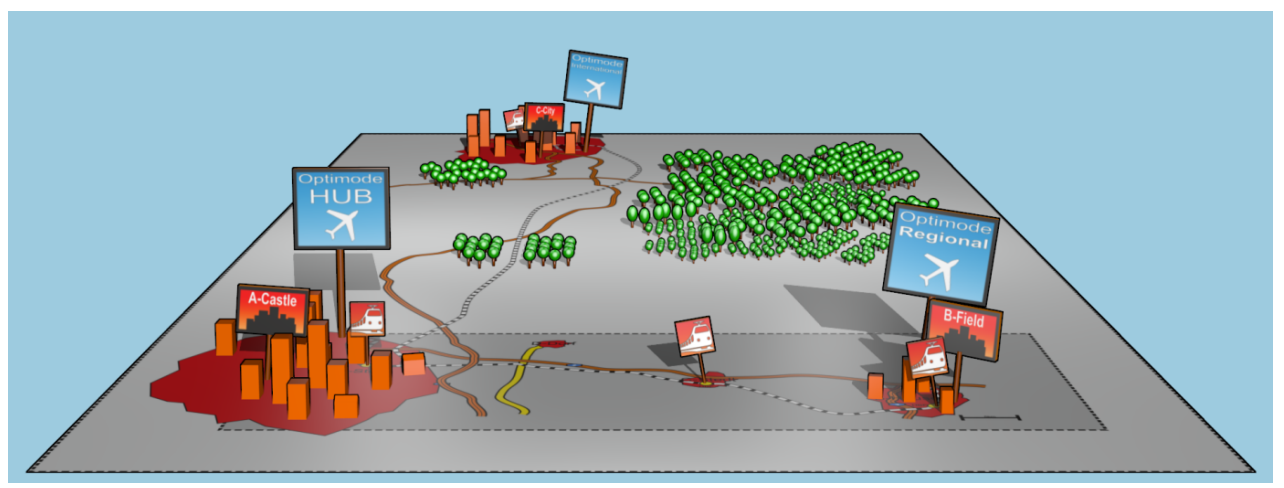

Figure 1: The virtual multi airport region of Optimode.net

The simulation model for the A-Castle airport can handle 40-60 million passengers per year, up to 400,000 flights and two terminals. The airport simulation of B-Field can handle 192,000 passengers and 35,000 flights per year. C-City airport has a passenger volume of approximately 16 million passengers per year distributed over some 160,000 flight movements.

To consider the relevant aspects for airport management different abstractions levels for the simulation are needed. From the airside sequences of take-offs and landings are sufficient. The in depth evaluation of in airport processes and measures depends on the individual simulation of persons in the airport (Figure 2). Individuals are created when arriving by aircraft or ground traffic.

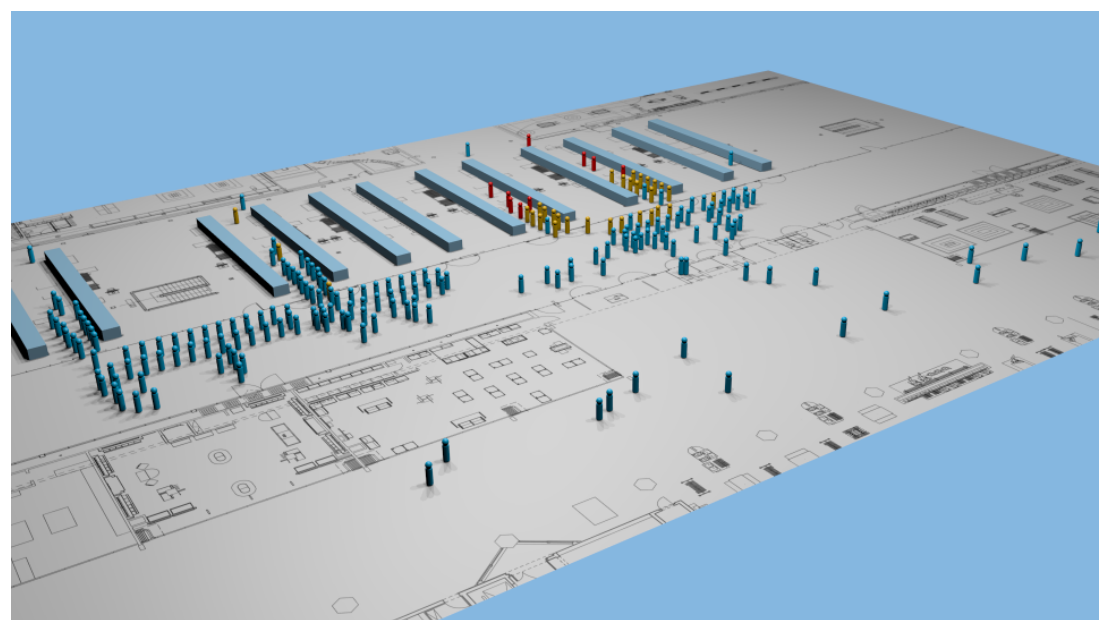

Figure 2: Simulation of security area (A-Castle)

Relevant public transport vehicles for the scenario are simulated with SUMO (Krajzewicz, Erdmann, Behrisch, \& Bieker, 2012) as part of the traffic combination to be coordinated by the traffic management. Public transport currently contains eight bus lines, six train and two tram connections with 1,035 cycles in 24 hours to connect the airports. The schedules for these connections are also based on real ones.

Regarding times, different reliabilities are needed for the handling and management of events and traffic participants. First, scheduled times are available for the whole day. As time progresses, estimated times differ from planned times and become more accurate. Finally, the actual time is available, as soon the relevant event occurs. 
These times for the simulated objects are the basis for examinations and measures of the airport management.

\subsection{Airport management}

The whole simulation setup is built to enable an (simulated) multi airport management control center access to information from all relevant traffic carriers. This integrated multimodal traffic management examined in the Optimode.net-project for different traffic carriers including air side is a novel approach, as it opens new scenarios to improve overall traffic and personal benefit for the individual passengers.

It is a quite common approach that a train waits for another late train to allow passengers catching their further connections. This waiting can be also seen for buses and partially for connections between trains and buses. However, including aircrafts into this integrated traffic management is a novel approach, which enables new possibilities. Time-consuming airport processes such as check-in and security checks also provide new potential for optimizations as selective prioritization for delayed passengers.

The airport management central presents an integrated view on all traffic participants to the human operator, who is doing his considerations in real time. The human operator is considered as final instance to assess the current situation. In addition, the operator has the possibility to re-plan the current schedules and influence the traffic events, for example, he can delay departure times. Figure 3 shows operators at work in the control center with Optimode.net.

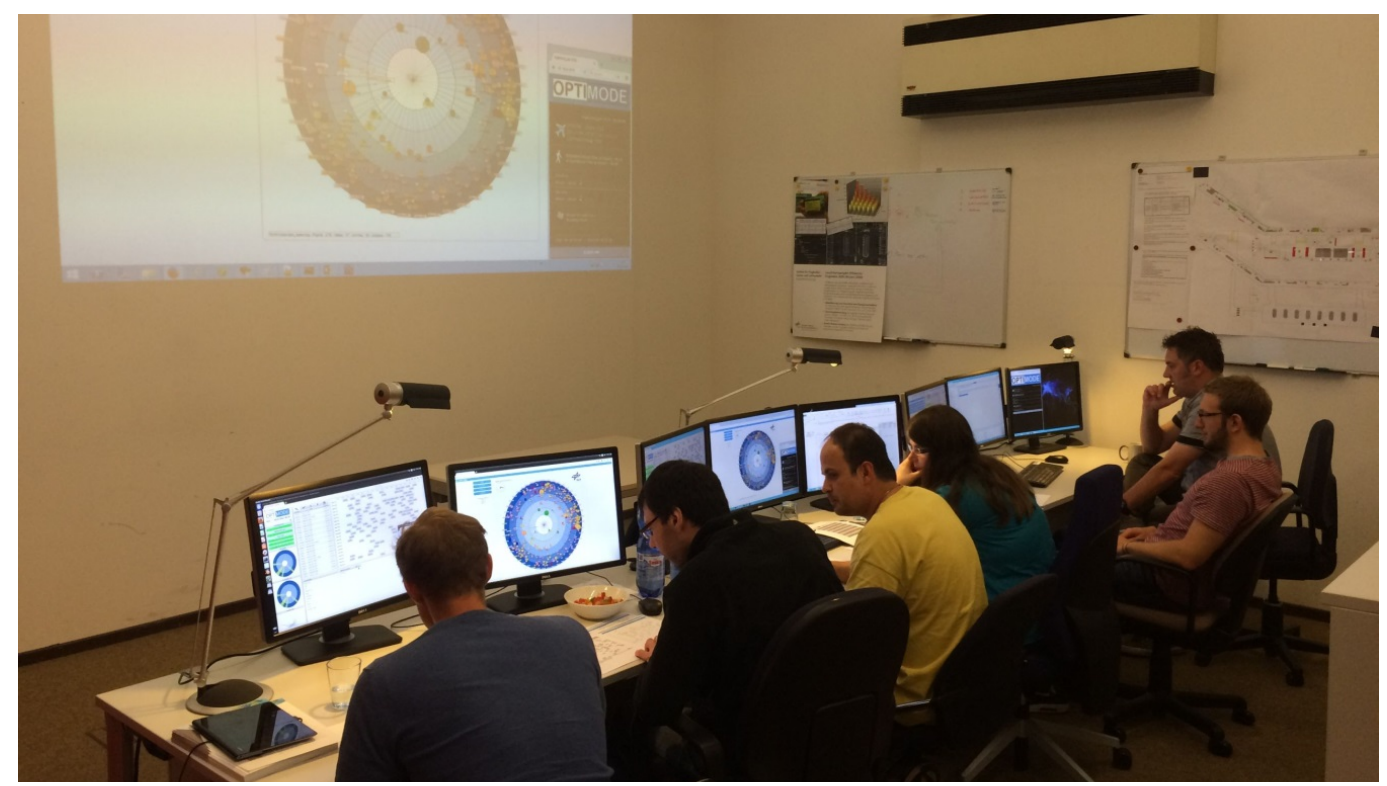

Figure 3: Airport management operators at work in a control center with Optimode.net

\subsection{What-if scenarios}

The airport multimodal transport hub is a very complex system, where little changes in the planning can have large and unforeseen impact. Because of this, the airport management operator needs support from the system for his decisions, which is provided in form of what-if scenarios. 
A what-if scenario gives the operator the possibility to fork the simulation of the current traffic situation and create a simulation of the nearby future with a modified planning. In this way, what-if scenarios provide the operator support and can present him, what impact his actions will likely have.

From a simulation perspective, what-if scenarios have quite strict requirements to the used simulation tools. The simulation tools must be able to continue their simulations from the current situation with two different scenarios, where changes are interactively performed during calculations. Furthermore, all simulation tools and the simulated scenarios must be synchronized. Details about the interaction between the tools are provided in the next section.

\section{Coupled simulation}

This section discusses the technical aspects of the coupled simulation. The system architecture combines relevant components and provides foundations for their interaction. For the coupling of simulations, the timing of their interaction is introduced. The last sub-section presents the detailed interaction with SUMO.

\subsection{System architecture}

The system architecture is divided into three main tool clusters as shown in Figure 4. The first tool cluster is the simulation environment. It contains the simulators for the railway system / public transport and simulators for each airport in the modeled cities. The airport simulators in each city are again differentiated in airport landside and airport airside.

The second tool cluster is the Optimode.net tool suite with the different tools that are needed for a proactive passenger management. The goal of the tool suite is to provide the operator with an early situational awareness, decision support and an efficient resource management.

The third tool cluster is the Integration Environment with the central MySQL Optimode.net database. This cluster cares for connecting the simulation environment with the tool suite as well as for synchronizing, exchanging results and for interactions within the overall simulation. The Optimode.net database contains all necessary master and dynamic data as well as system parameters and resulting data of the single modules. Master data is a fixed set of data that will not be modified during a system run and contains e.g. details about worldwide airports, airlines and aircraft. 


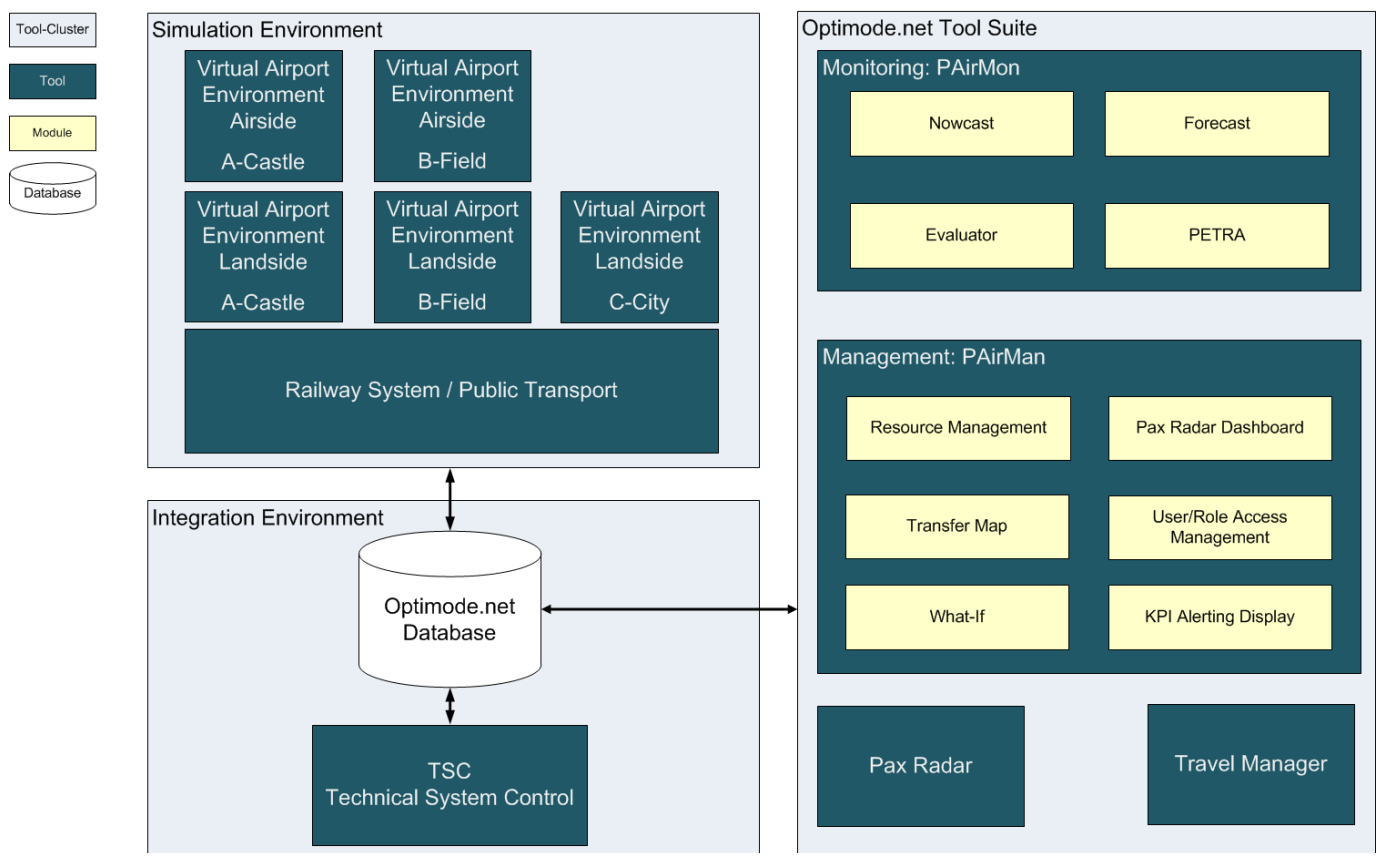

Figure 4: Optimode.net system architecture

The dynamic data is structured in scenarios that specify the behavior of a system run. A scenario is described by its schedules for flights, public ground transport and process points. Additional information is provided concerning in- and outbound connections of transfer passengers. As process points must be operated by personnel, thus human resources pool is also part of the database. The "Technical System Control Tool" controls information about the active system run and creates also timestamps to synchronize the system modules. Each single modification of the schedule is tracked to allow a detailed reconstruction of events and their impacts on the entire system. For more technical details see (Jung, Rudolph, Claßen, Pick, \& Noyer, 2017).

\subsection{Coupling of simulations}

Figure 5 shows the different simulation models underlying the "Passenger Information System". For each user, the simulation environment computes milestones for their personal travel chain with differing characteristics. The first milestone computed by the simulation environment is the OPHT (Outbound Passenger at HBF Time) which represents the time value when the passenger enters the main station (HBF). The following table explains the presented milestones in Figure 5 in chronological order. 


\begin{tabular}{|c|c|c|}
\hline Milestone & Description & Simulation \\
\hline $\begin{array}{l}\text { OPHT (Outbound Passenger } \\
\text { at HBF time) }\end{array}$ & $\begin{array}{l}\text { Passenger enters the main } \\
\text { station }\end{array}$ & $\begin{array}{l}\text { Calculated values based on } \\
\text { public transport }\end{array}$ \\
\hline $\begin{array}{l}\text { OCHT (Outbound Checked } \\
\text { at HBF time) }\end{array}$ & $\begin{array}{l}\text { Passenger leaves the main } \\
\text { station and enters the train }\end{array}$ & $\begin{array}{l}\text { Scheduled values based on } \\
\text { public transport and actual } \\
\text { values computed by SUMO }\end{array}$ \\
\hline $\begin{array}{l}\text { OPFT (Outbound Passenger } \\
\text { at Airport Railstation Time) }\end{array}$ & $\begin{array}{l}\text { Passenger enters the airport } \\
\text { railstation }\end{array}$ & $\begin{array}{l}\text { Scheduled values based on } \\
\text { Public transport and actual } \\
\text { values computed by SUMO }\end{array}$ \\
\hline $\begin{array}{l}\text { OCFT (Outbound Checked } \\
\text { at Airport Railstation Time) }\end{array}$ & $\begin{array}{l}\text { Passenger leaves the airport } \\
\text { railstation }\end{array}$ & $\begin{array}{l}\text { Calculated values based on } \\
\text { public transport }\end{array}$ \\
\hline $\begin{array}{l}\text { OPAT (Outbound Passenger } \\
\text { at Airport Time) }\end{array}$ & Passenger enters the airport & $\begin{array}{l}\text { Calculated values based on } \\
\text { public transport }\end{array}$ \\
\hline $\begin{array}{l}\text { OPCT (Outbound Passenger } \\
\text { at Check In Time) }\end{array}$ & Passenger reaches the check in & Calculated values \\
\hline $\begin{array}{l}\text { OCCT (Outbound Checked } \\
\text { at Check In Time) }\end{array}$ & Passenger is checked in & $\begin{array}{l}\text { Estimated values based on } \\
\text { forecast simulation, actual } \\
\text { values based on reality } \\
\text { simulation }\end{array}$ \\
\hline $\begin{array}{l}\text { OPST (Outbound Passenger } \\
\text { at Security Time) }\end{array}$ & Passenger reaches the security & $\begin{array}{l}\text { Actual values based on reality } \\
\text { simulation }\end{array}$ \\
\hline $\begin{array}{l}\text { OCST (Outbound Checked } \\
\text { at Security Time) }\end{array}$ & Passenger is security checked & $\begin{array}{l}\text { Estimated values based on } \\
\text { forecast simulation, actual } \\
\text { values based on reality } \\
\text { simulation }\end{array}$ \\
\hline $\begin{array}{l}\text { OPET (Outbound Passenger } \\
\text { at Emigration Time) }\end{array}$ & $\begin{array}{l}\text { Passenger reaches the } \\
\text { emigration }\end{array}$ & Calculated values \\
\hline $\begin{array}{l}\text { OCET (Outbound Checked } \\
\text { at Emigration Time) }\end{array}$ & $\begin{array}{l}\text { Passenger passes the } \\
\text { emigration }\end{array}$ & $\begin{array}{l}\text { Estimated values based on } \\
\text { forecast simulation, actual } \\
\text { values based on reality } \\
\text { simulation }\end{array}$ \\
\hline $\begin{array}{l}\text { OPGT (Outbound Passenger } \\
\text { at Gate Time) }\end{array}$ & Passenger reaches his gate & $\begin{array}{l}\text { Calculated and estimated } \\
\text { values based on forecast } \\
\text { simulation }\end{array}$ \\
\hline $\begin{array}{l}\text { OCGT (Outbound Checked } \\
\text { at Gate Time) }\end{array}$ & $\begin{array}{l}\text { Passenger leaves the airport } \\
\text { and enters the aircraft }\end{array}$ & $\begin{array}{l}\text { Estimated values based on } \\
\text { forecast simulation, actual } \\
\text { values based on reality } \\
\text { simulation and airside } \\
\text { simulation (TOP) }\end{array}$ \\
\hline
\end{tabular}

Milestones computed by SUMO are OCHT (Outbound Checked at HBF Time), it represents the time value the passenger enters the train to the airport, and OPFT (Outbound Passenger at Airport Railstation Time), this milestone represents the time value the passenger leaves the train and enters the airport railstation. The OPFT (Outbound Passenger at Airport Railstation Time), this milestone represents the time value the passenger leaves the train and enters the airport railstation.

When the passenger enters the airport (OPAT - Outbound Passenger at Airport Time) it will be simulated by the Passenger Movement Reality Simulation (Figure 2). The movement simulation 
comes to an end, when the passenger enters the aircraft (OCGT - Outbound Passenger Checked at Gate Time). The airside simulation (TOP) calculates this final outbound milestone.

These characteristics follow the SCETA ( $\underline{\boldsymbol{S}}$ cheduled, $\underline{\boldsymbol{C}}$ alculated, $\underline{E}$ stimated, $\underline{T}$ arget, $\underline{\boldsymbol{A}}$ ctual) model and range from planned times derived from the flight schedules (scheduled), through calculated times. The calculated times take into account the current traffic situation (calculated), as well as expected times (estimated), which incorporate the first confirmations of the passenger's preceding nodes, up to the target times (target), which define, within their characteristics, the specific point in time which is to be effectively achieved. Finally, the actual measured timestamp (actual), which then lies in the past. A prototypical application with the applied milestones is presented by (Milbredt, Rudolph, \& Grunewald, 2016).

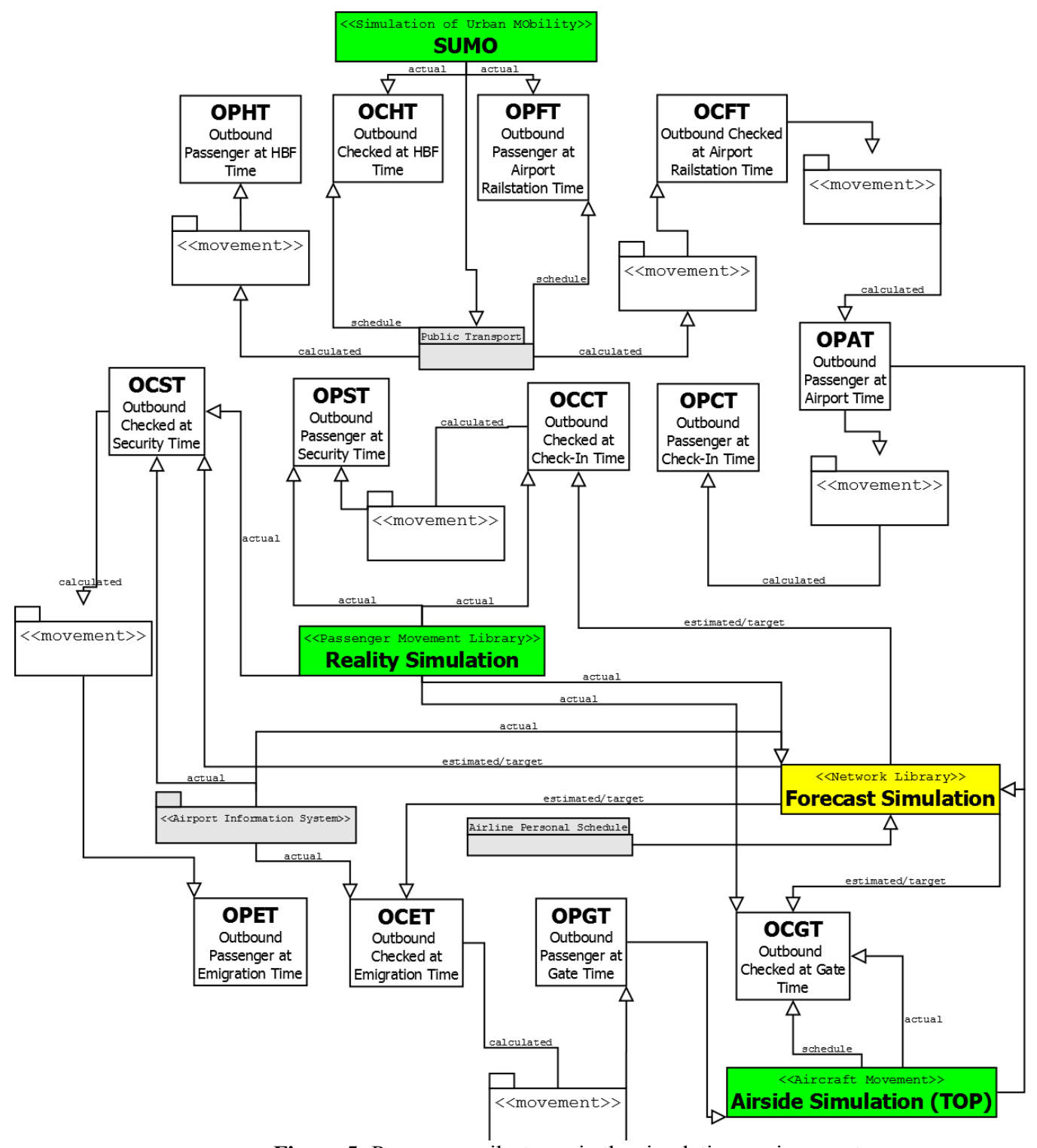

Figure 5: Passenger milestones in the simulation environment 


\subsection{Interaction with SUMO}

The public transport simulation is realized using SUMO and covers the simulation of ground level public transportation to model incoming and outgoing passengers by buses and trains to and from the airports. Furthermore, as a regional area is considered in the simulation scenario, a train could be considered to replace a feeder flight between the local airports under certain circumstances to analyze the impacts.

SUMO was chosen, because it is an open source microscopic traffic simulation, which provides information about individual vehicles at any time including buses and trains. Furthermore, it is highly configurable and interactions and reconfigurations are possible to manipulate the simulation as needed for the interaction with the airport management operator and to realize the what-if scenarios.

For communication with the Optimode.net environment, the Optimode.net database contains all the scheduled public transport connections. Besides the scheduled time for the connection, the estimated, targeted and actual times are foreseen and provided when available. Same data must be handled for each what-if scenario.

SUMO can be purely configured by files and run from the command line, what makes it perfect for scripting and an automated interaction with the Optimode.net environment. Furthermore, an API is available to interact with the simulation. The API was examined during development but not used, as the command line interface is more suitable for this project, because saving and resuming simulation states is more suitable for the described use case. SUMO is purely configured by a set of XML files and generates the output as XML files as well.

For Optimode.net a dedicated component "Optimode.net-SUMO-Adapter" was implemented to run SUMO as required by the entire simulation as well as to feed SUMO with the right input and write results back into the shared Optimode.net database. The input files for SUMO include e.g. the traffic network and the vehicles with their routes and scheduled stops. Furthermore, the simulation generates the actual performed stops of the vehicles as output and some other information. These dependencies are shown in Figure 6.

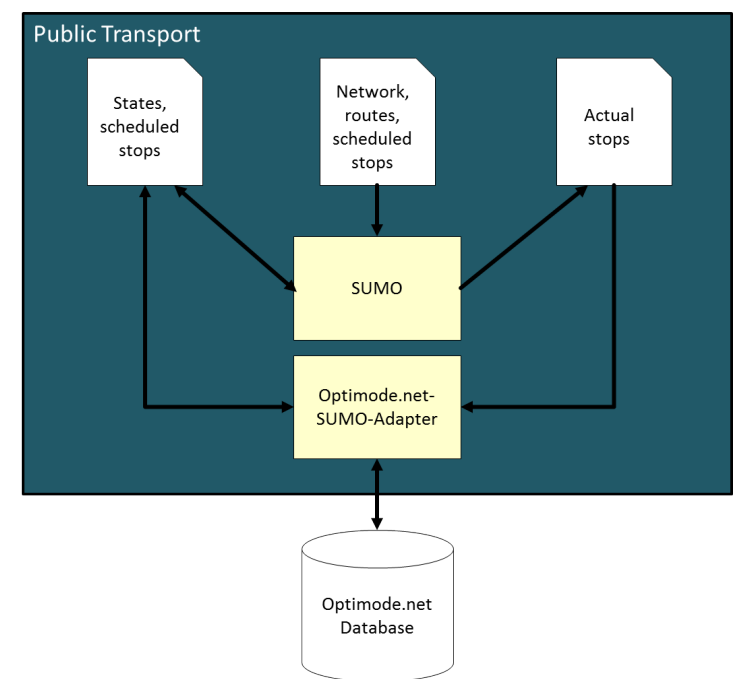

Figure 6: Interaction between SUMO and Optimode.net

The management center visualizes the current traffic state and is updated every minute in simulated time. This update frequency is perfectly valid as traffic management centers also only have 
data available with some minor delay in reality. The simulated time is triggered by a dedicated time generator, which provides the actual time in the database.

In the background SUMO is always started each time to simulate the next minute and save the final state to the Optimode.net environment, using --save-state.times and --savestate.files parameters. Furthermore, SUMO continues simulation for a fixed simulated time (i.e. 30 minutes) to calculate estimations for this possible future. When the management center requests the next update, SUMO re-starts from the last saved state to create the next saved state and calculate an updated forecast, what is done repeatedly. For this approach, SUMOs feature to create save states and continue simulation from them is heavily used. Furthermore, for this application SUMO must be 30 times faster than simulated real time.

In case of a request by the virtual airport management to adapt the target time of a public transport connection, this can be easily applied by the SUMO adapter. If the vehicle is not yet started, just the scheduled stops are adapted and the simulation is continued. If the vehicle has already joined the simulation, the saved state file of the current state is adapted accordingly (see Figure 6). Finally, the adapter synchronizes the actually performed stops of relevant vehicles from the SUMO files with the database after each simulation cycle.

As discussed in section 2.3 the simulation must also support what-if scenarios. Technically this means, it must be possible to fork the simulation from its current state into two different simulations with different parameters. Using saved states, this is possible very easily. This means, that just a copy of a saved state has to be created and adapted to the requests from the management center. As states and all other input and output files are available in XML this step is quite easy. It is just necessary to consider, that as soon a vehicle has entered the simulation, its scheduled stops move to the state file and become output and input again for the simulation.

\section{Summary and outlook}

This paper discusses the simulation of traffic and passengers to reproduce a multi airport region from the perspective of an airport traffic management, where the airport has the role of a central transport hub. To reach this goal several simulators are coupled, which cover different traffic modes and roles in this complex environment. Finally, a visualization and interaction for the virtual airport traffic management center is provided.

Furthermore, a scalable system architecture is introduced, which provides the foundations to couple the relevant simulations and integrate several individual components. Only by the separation of concerns in manageable sub-modules, the whole system keeps also manageable.

In this compound system, SUMO has the important role to simulate the ground level public transport including buses and trains. For this purpose, SUMO's outstanding capabilities to interact with the simulation and adapt details of simulation states are needed. Even more, SUMO can fork the simulation to continue from a simulation state with differently parametrized simulations, what is needed to realize what-if scenarios and provide decision support for the traffic management.

The sub-systems are based on real data. Their behavior is essentially known and adequately modelled. Examinations become interesting and new on top of the coupling of the dedicated systems. Here, new traffic management questions and measures can be investigated, which involve interactions between the airport and other traffic modes.

Currently the project Optimode.net has reached a state, where the coupled simulation is successfully running. The finalization of what-if scenarios will be realized during the next month. Based on these foundations, specific validation scenarios will be compared with and without the Optimode.net management system. First tests run with DLR personnel and further examinations will be performed with real life airport practitioners from different stakeholders. 


\section{References}

Eurocontrol. (2012, March). Airport CDM Implementation - The Manual. Brussels.

Jung, M., Rudolph, F., Claßen, A. B., Pick, A., \& Noyer, U. (2017). Simulating a multi-airport region to foster individual door-to-door travel. Winter Simulation Conference Proceedings ( $\mathrm{pp}$. 2518 - 2529). Las Vegas: Omnipress.

Krajzewicz, D., Erdmann, J., Behrisch, M., \& Bieker, L. (2012). Recent Development and Applications of SUMO - Simulation of Urban MObility. International Journal On Advances in Systems and Measurements, pp. 128-138.

Milbredt, O., Rudolph, F., \& Grunewald, E. (2016). Passenger-centric Intermodal Traffic Management involving Airports and Railways. Canadian Transportation Research Forum CTRF 51 st Annual Conference. Toronto, Canada.

Milbredt, O., Rudolph, F., Grunewald, E., \& Christ, T. (2017). Evaluating conditions and impact of intermodal traffic management involving airports and railways. Transportation Research Procedia (pp. 1740 - 1749). Hongkong: Elsevier. 